\title{
ESTUDIO QUIIMICO-MINERALÓGICO DE LOS PERFILES LATERÍTICOS FERROSIALÍTICOS EN LOS SECTORES TÉNEME, FARALLONES Y CAYO GUAM EN EL NORESTE DE CUBA
}

\author{
CHEMICAL-MINERALOGICAL STUDY OF THE FERROSIALITIC \\ LATERITIC PROFILES IN THE TENEME, FARALLONES AND CAYO GUAM \\ SECTORS IN NORTH EAST CUBA
}

\author{
Tendai Njila ${ }^{1}$ \& Roberto Díaz-Martínez ${ }^{2}$ \\ ${ }^{1}$ Department of Geology, University of Zimbabwe. Box MP167, Mount \\ Pleasant, Harare, Zimbabwe \\ ${ }^{2}$ Profesor Titular. Toluca, México \\ *Autor para contacto: drtnjila@gmail.com
}

(Recibido: 20/12/2014; aceptado: 22/02/2016)

\begin{abstract}
The results of a chemical-mineralogical study on the ferrosialitic lateritic weathering profiles are presented. Analysis was done using X Ray Fluorescence and Diffraction. The chemical composition of these profiles reflects a typical pattern normally observed within such weathering crusts. The sesquioxides of aluminium, iron are concentrated in the upper parts of the profiles as a result of the leaching of alkalis, alkaline-earth elements, and to a lesser extent, silica. Ferrosialitic lateritic profiles formed on Cretaceous and Paleogene islands arcs consist of secondary minerals such as gibbsite, kaolinite, goethite, hematite and montmorillonite, while those associated with basic and volcanogenicsedimentary rocks of ophiolitic affinity in Cayo Guam are composed of gibbsite and iron oxides. This study brings new chemical-mineralogical information on the ferrosialitic lateritic profiles associated with rocks of oceanic nature in north-eastern Cuba, which will help in the exploration for industrial minerals, and rocks and their application in industry. Keywords: Ferrosialitic, laterites, weathering profiles, Cuba.
\end{abstract}

\footnotetext{
Resumen: Se presentan los resultados de un estudio químico-mineralógico de los perfiles lateríticos ferrosialíticos sobre rocas básicas de naturaleza oceánica y las litologías propias de los arcos de islas en la región nororiental de Cuba. El procedimiento contempló el muestreo de cinco perfiles de alteración y el análisis mediante la Fluorescencia y Difracción de Rayos X. Los perfiles lateríticos ferrosialíticos originados a partir de rocas pertenecientes a los arcos de islas cretácico y paleógeno están constituidos por minerales secundarios que son gibbsita, caolinita, goethita, hematita y montmorillonita, mientras que los asociados a rocas básicas y vulcanogeno-sedimentarios de afinidad ofiolítica concentran gibbsita en Cayo Guam. Se aporta nuevos conocimientos químico-mineralógicos sobre los perfiles lateríticos ferrosialíticos asociados a rocas de naturaleza oceánica en Cuba nororiental, lo que ayudará en la exploración para rocas y minerales industriales y su aplicación en la industria.

Palabras claves: ferrosialíticos, lateritas, meteorización, Cuba.
} 


\section{INTRODUCCIÓN}

La insuficiente caracterización desde el punto de vista químico-mineralógico de los perfiles lateríticos ferrosialíticos en los sectores Téneme, Farallones y Cayo Guam, no permite esclarecer la formación, clasificación y aplicabilidad industrial de estos objetos geológicos.

El estudio, por primera vez, de la composición química y mineralógica de los perfiles de meteorización ferrosialíticos en los sectores Téneme, Farallones y Cayo Guam en el noreste de Cuba a través de sus perfiles de alteración típicos, es un tema de absoluta novedad y actualidad en el escenario geológico mundial, a nivel nacional y muy particularmente en esta región nororiental de la isla.

Estos perfiles de meteorización se relacionan genéticamente con cortezas lateríticas que se han formado a partir de la descomposición de rocas de naturaleza oceánica (gabros y basaltos de la asociación ofiolítica) y rocas pertenecientes a los arcos del Cretácico (basaltos, andesitas, andesitabasaltos y dacitas) y del Paleógeno (tobas y tufitas); por tanto, cualquier intento de estudio químico-mineralógico de los perfiles ferrosialíticos en Cuba necesariamente tiene que incluir estos tipos de litologías, pues hasta entonces solamente se le prestaba atención a las cortezas desarrolladas sobre gabros y calizas, que hasta estos momentos eran las únicas que disponían de estudios similares a los presentados en esta investigación.

Se esclarece la composición química y mineralógica de los diferentes perfiles lateríticos en los sectores estudiados con la finalidad de clasificarlos desde el punto de vista químico y mineralógico. Además, se establece el balance de meteorización en los perfiles lateríticos analizados para determinar el grado de madurez y zonalidad químico-mineralógica.

La aplicación práctica de los resultados obtenidos radica en la posible utilización de las arcillas asociadas a perfiles ferrosialíticos en la industria cerámica, refractaria y materiales de la construcción.

\section{Características físico-geográficas y geológicas de la región}

El área de estudio está ubicada entre Frank País y Moa (Figura 1) definido por las coordenadas de Lambert siguientes: X: 660000 - 720 000; Y: $200000-220000$. La región de estudio tiene un relieve predominantemente montañoso hacia la parte sur (grupo montañoso Sagua-Baracoa) debido a la presencia de la Sierra de Moa que se extiende en dirección submeridional.

El clima es tropical con abundantes precipitaciones anuales que oscilan entre $1600-2200$ mm y la evaporación anual entre $2200-2400$ mm (Oliva, 1989). La red fluvial es densa y de tipo dendrítica, representada por numerosos ríos y arroyos (Levisa, Cabonico, Téneme, Grande, Sagua, Cabañas, Moa, Cayo Guam, etc.), que mantienen un buen caudal todo el año. La vegetación comprende el $33 \%$ del endemismo cubano.

\section{Marco geológico regional}

La geología de la región se caracteriza por una marcada complejidad, condicionada por un variado mosaico de litologías y diversos eventos tectónicos ocurridos en el transcurso del tiempo geológico, lo que justifica los diferentes estudios geológicos regionales y locales. Las cortezas de meteorización estudiadas están estrechamente relacionadas con diferentes formaciones geológicas.

\section{Ofiolitas septentrionales}

Las rocas ofiolíticas típicas están ampliamente representadas en las áreas de estudio, formando parte del macizo Sagua-Moa-Baracoa. De mayor interés en este trabajo son las litologías propias del complejo de acumulados básicos pertenecientes a la corteza oceánica representadas por extensos cuerpos y diques de gabroides con rasgos propios de gabros bandeados en la base del complejo y 


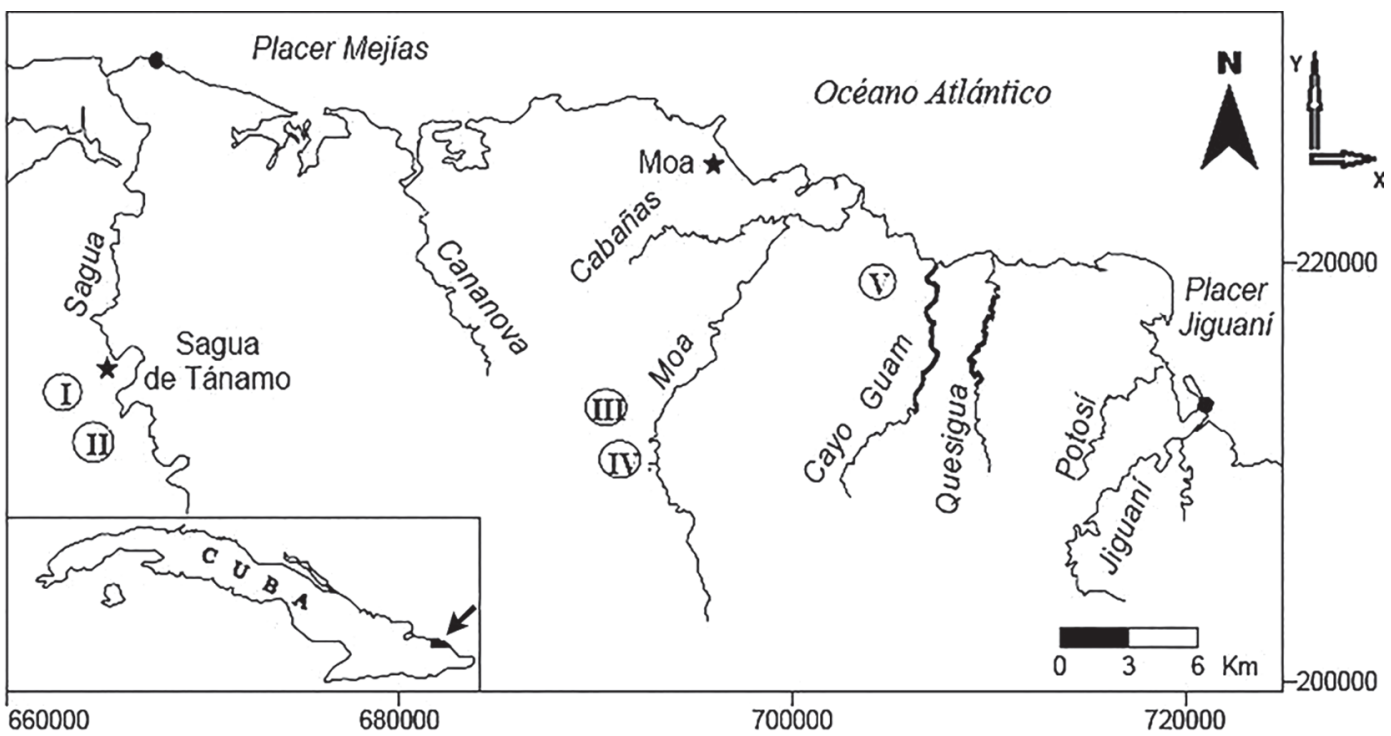

Fig. 1: Ubicación geográfica de los sectores de estudio. I-Baconal; II-El Culebro; III-Caimanes; IV-Farallones; V-Cayo Guam.

hacia la parte superior, con textura propia de los gabros isotrópicos (Ríos \& Cobiella, 1984; Fonseca et al., 1985).

\section{Formación Téneme}

Las rocas volcánicas son principalmente basaltos, andesitas basálticas, andesitas, y en menor grado, dacitas. Su composición química varía entre toleitas de arco de islas de afinidad ofiolítica con bajos contenido de titanio, de tipo N-MORB y toleitas típicas de arco de islas oceánicas. Los basaltos muestran relaciones bajas de LREE/Yb $(\mathrm{La} / \mathrm{Yb}<$ 5), típicas de arcos de islas interoceánicos. Estos y otros datos geoquímicos advierten que las rocas pertenecientes a esta formación pudieran ser parte de un antiguo arco de isla tipo PIA (Proenza et al., 2006).

\section{Formación Sabaneta}

Estas secuencias están compuestas por tobas vitroclásticas, litovitroclásticas, cristalovitroclásticas con intercalaciones de tufitas calcáreas, areniscas tobáceas, calizas, conglomerados tobáceos, lutitas, margas, gravelitas, conglomerados volcanomícticos y algunos cuerpos de basaltos, andesitas, y andesitas-basálticas, los cuales alcanzan hasta $6000 \mathrm{~m}$ de espesor (Cobiella, 1978; Proenza \& Carralero, 1994; Iturralde-Vinent, 1996, 1998).

\section{MATERIALES Y MÉTODOS}

\section{Recopilación y procesamiento de la información existente}

Se consultaron numerosos trabajos publicados hasta el año 2010, relacionados principalmente con la composición química y mineralógica de las cortezas ferrosialíticas, factores de la meteorización química, zonalidad geoquímica de los elementos químicos, clasificación, el balance de la meteorización y las posibles aplicaciones industriales en base a la composición química y mineralógica, partiendo desde a nivel mundial hasta a nivel de la región de estudio.

Se consultaron informes técnicos de la Oficina Nacional de Recursos Minerales (ONRM), publicaciones científicas nacionales e internacionales, 
trabajos presentados en eventos y congresos, así como trabajos de diploma, tesis de maestría y doctorales, realizados hasta el año 2010.

En Cuba, partiendo del año 1943 se han realizado investigaciones intensas en busca de bauxitas (Nagy, 1976, 1983; Lavandero et al., 2009), pero estas no determinaron la presencia de algún depósito económicamente explotable.

Varios autores e instituciones han caracterizado desde diferentes puntos de vista geológicos el depósito de bauxitas más importante en Cuba, "Sierra Azul - Pan de Guajaibón" en Pinar del Río (Formell \& Buguelskey, 1984; Hernández, 1989; Hernández \& Hernández, 1985).

En Ciego de Ávila y Camagüey los cuerpos bauxíticos y alíticos se caracterizan por presentar contenidos altos de los elementos itrio, iterbio, lantano y escandio, algunos de los cuales pueden tener valores industriales significativos, sobre todo para la explotación conjunta con las bauxitas.

Muchos investigadores han trabajado en las cortezas de meteorización no ferroniquelíferas con el objetivo de encontrar y evaluar bauxitas (Guerasimov et al., 1976; Elmer, 1977; Formell et al., 1977; Coutin et al., 1981; Ponce et al., 1985; Orozco, 1995; Pons \& Leyva, 1996; Fonseca et al., 2004).

Además, se realizaron varios trabajos de diploma relacionadas con la evaluación de las perspectivas bauxíticas y las cortezas de meteorización no ferroniquelíferas en los diferentes sectores de las Provincias Holguín y Guantánamo entre 1980 y 2010 (González \& Bárcaz, 1981; Cobas \& Vargas, 1982; De Dios \& Cueto, 1982; Moreno \& Pérez, 1982; Pino \& Rosales, 1982; García, 1985; González, 1991; Betancourt, 1992; Oliva, 1992; Fadel, 2005; Cámara, 2007; Perdomo, 2008). El objetivo era encontrar las bauxitas pero tampoco se pudo confirmar la existencia de bauxitas sensu strictus. De igual manera, no se realizaron evaluaciones de las perspectivas industriales de materiales ricos en alúmina existentes en esta región.

Estudios más recientes (Fadel, 2005; Cámara, 2007; Perdomo, 2008; Lavandero, 2009; Njila et al., 2010) en Cuba Oriental fundamentan la importancia de continuar las investigaciones en dichas cortezas desarrolladas sobre litologías no ultrabásicas, como son las rocas gabroides, vulcanitas y metavulcanitas del arco del Cretácico, rocas volcánicas y vulcanógeno-sedimentarias dístales del arco del Paleógeno.

Algunos estudios señalan (Guerasimov et al., 1976; Nagy, 1976, 1983) que no es posible encontrar grandes depósitos de bauxita tipo Jamaica en Cuba Sin embargo, no se evaluaron las posibilidades de encontrar las manifestaciones ricas en aluminio que podrían explotarse económicamente $\mathrm{y}$ tener importantes usos industriales.

\section{Selección de perfiles}

Los trabajos de campo se realizaron en los sectores de Baconal y El Culebro y los sectores Caimanes, Farallones y Cayo Guam (Cuadro 1). Estos trabajos tuvieron como objetivo principal obtener muestras a todo lo largo de 5 perfiles de meteorización representativos por el método de surco continuo.

\section{Fundamentación de la selección de los perfiles lateríticos en los sectores de estudio}

La selección de los perfiles lateríticos en los sectores Téneme, Farallones y Cayo Guam se fundamentan en los siguientes factores: (a) la insuficiencia de la información química y mineralógica de potentes cortezas de meteorización con típicos perfiles lateríticos resultantes de la meteorización de rocas básicas (gabroides) de la asociación ofiolítica y de las rocas volcánicas y vulcanógeno-sedimentarias de los arcos de islas del Cretácico y del Paleógeno en los sectores de estudio, (b) la buena accesibilidad a los tres sectores de estudio, (c) la explotación limitada y sin un control de la calidad del mineral en un pequeño tejar en Centeno, (d) amplias posibilidades de la utilidad de los recursos estudiados en el desarrollo endógeno de los territorios del noreste de la provincia de Holguín, y (e) los espesores de los perfiles en 
Cuadro 1

Ubicación de las muestras recolectadas por sectores de estudio

\begin{tabular}{cccccc}
\hline \multirow{2}{*}{ Municipio } & Sector & \multicolumn{2}{c}{ Coordenadas } & \multirow{2}{*}{$\begin{array}{c}\text { Punto de } \\
\text { muestreo }\end{array}$} \\
\cline { 3 - 4 } & & $\mathrm{X}$ & $\mathrm{Y}$ & & Muestras \\
\hline Frank & Baconal & 665852 & 216442 & 4 & TNL-1, TNL-2, TNL-3 \\
País & El Culebro & 667276 & 215839 & 5 & TNL-4,TNL-5, TNL-6, TNL-7 \\
Moa & Caimanes & 687834 & 211936 & 6 & TNL-8, TNL-9, TNL-10, TNL-11, TNL-12 \\
& Farallones & 690611 & 207108 & 7 & TNL-13, TNL-14, TNL-15, TNL-16 \\
& Cayo Guam & 706600 & 217940 & 8 & CG-1, CG-2, CG-3, CG-4, CG-5 \\
\hline
\end{tabular}

los sectores seleccionados son mayores a 10 metros, tienen buena aflorabilidad y su extensión es más de $15 \mathrm{~km}^{2}$.

\section{Documentación y muestreo de los perfiles}

Se seleccionaron los perfiles más representativos y las muestras se tomaron por el método de surco.

\section{Trabajos de laboratorio}

\section{Preparación de las muestras y separación granulométrica}

Las muestras fueron secadas al sol, pesadas, homogenizadas, pesadas de nuevo, cuarteadas y tamizadas.

\section{Medición del pH}

La medición del $\mathrm{pH}$ se realizó en el Laboratorio de Química del ISMM empleando el método titrimétrico. Se pesaron 10 gramos de cada muestra con la balanza eléctrica. Después se colocaron en un beaker y se le añadieron $100 \mathrm{ml}$ de agua destilada usando un measuring cylinder. Se colocó el beaker sobre el agitador eléctrico hasta que la muestra se mezcló totalmente. Luego se instalaron los electrodos en la solución para medir el $\mathrm{pH}$ usando el Titrador. La lectura en la pantalla del equipo se descargó a la computadora para ser procesado en el Microsoft Excel.

Análisis químico de roca total por Fluorescencia de Rayos X (FRX)

Los análisis químicos de roca total se realizaron mediante la técnica analítica FRX en la Universidad de Barcelona (UB), España, empleando un espectrómetro de marca Panalytical (Philips) PW2400 con un tubo $\mathrm{Rh}$ de rayos X operado a $60 \mathrm{KeV}, 125 \mathrm{~mA}$ y $3000 \mathrm{~W}$, y en la Empresa Geominera Oriente, Cuba, usando un espectrómetro Philips PW2400.

Análisis mineralógico por Difracción de rayos $X$ (DRX)

Se determinó la composición mineralógica cualitativa de las muestras mediante difracción de rayos $\mathrm{X}(\mathrm{DRX})$ en la Universidad Técnica de Claustral (TUC), Alemania, empleando un difractómetro alemán tipo $\mathrm{HZG}$ - 4 con radiación de CoK $\alpha$ y velocidad del goniómetro de $2 \%$ min.

La interpretación cualitativa de los difractogramas se realizó con ayuda del software ANALYZE, de la firma Seifert, Alemania, mientras los análisis cuantitativos se realizaron con el software AUTOQUAN, de la misma firma y que emplea el método Rietveld para el ajuste de las curvas entre los patrones seleccionados y la muestra investigada. 


\section{Índices Químico-Mineralógicos}

Los índices de meteorización química son ecuaciones que, sobre la base del análisis químico de las rocas, permiten conocer el grado de descomposición o la medida de la cantidad de meteorización que ha tenido lugar. El cálculo de los índices se basa en las relaciones entre los óxidos menos móviles (esencialmente $\mathrm{Al}_{2} \mathrm{O}_{3}, \mathrm{TiO}_{2}$ ) y los más móviles o solubles en condiciones superficiales $\left(\mathrm{CaO}, \mathrm{Na}_{2} \mathrm{O}, \mathrm{K}_{2} \mathrm{O}\right)$.

Los índices de meteorización química empleados en este trabajo son: Índice Residual de Vogt (V; Vogt, 1927 y Roaldset, 1972), Factor de Lavado (FL; Jenny, 1941), Índice de Meteorización de Reiche y Vogel (MWPI; Reiche, 1943 y Vogel, 1975), Índice de Meteorización Potencial (WPI; Reiche, 1950), Proporción de Ruxton (R; Ruxton, 1968), Índice de Meteorización de Parker (WI o WIP; Parker, 1970), Índice de Englund y Jorgensen (M1, M2; Englund y Jorgensen, 1973), Índice Químico de Alteración (CIA; Nesbitt y Young, 1982), El Índice Químico de Meteorización (CIW; Harnois, 1988), Índice de Alteración de Plagioclasas (PIA; Fedo et al., 1995), e Índice de Móviles (Imob; Irfan, 1996). Todas las siglas están en inglés.

La información detallada sobre los índices mencionados se puede encontrar en las referencias citadas. Estos índices son esencialmente correlaciones de la composición química de las cortezas en los perfiles de alteración y sus materiales originales inalterados, principalmente de los elementos móviles (álcalis, alcalino-térreos, sílice) y los no o poco móviles (circonio, titanio, aluminio).

El índice químico-mineralógico analizado en este trabajo es el Índice de Alteración Mineralógica (MIA) (Voicu et al., 1997).

\section{Clasificaciones química y químico-mineralógi- ca de las cortezas de meteorización}

La clasificación química de las cortezas de meteorización se basó en su composición química tomando las relaciones entre los componentes principales aluminio, hierro y sílice que se presentan en un diagrama ternario, tomando como criterio un contenido de alúmina mayor que $20 \%$ en peso.

Además, se empleó el Índice de Alitización Potencial (API) para la clasificación química de los perfiles de meteorización ferrosialíticos en base a su composición química y la escala vertical de intensidad de alteración.

\section{RESULTADOS Y DISCUSION}

\section{Distribución granulométrica}

Las fracciones granulométricas se definieron como: finas $(<0,063 \mathrm{~mm})$, intermedias $(>0,063<0,18 \mathrm{~mm})$ y gruesas $(>0,18 \mathrm{~mm})$ para todos los sectores estudiados.

En Baconal las fracciones finas e intermedias predominan en las partes superiores del perfil, las fracciones finas ocupan entre 59,2 y $62,8 \%$ de la muestra en los horizontes superiores e intermedios, respectivamente, mientras que las fracciones intermedias llegan a valores de 25,6 y 24,64 \% en dichos horizontes. La fracción gruesa es menos representativa en estos horizontes que en la parte inferior del perfil, donde predomina con valores superiores a $90 \%$.

En el sector El Culebro, la fracción fina predomina en todo el perfil con un promedio de $70,39 \%$. La fracción intermedia aumenta en las partes intermedias e inferiores, pero su valor es muy bajo en la parte superior del perfil. Los valores de la fracción gruesa son extremadamente bajos en la parte intermedia hacia la base del perfil y esta fracción solo aparece en cantidades pequeñas entre 13 y $17 \%$ en los horizontes superiores e intermedios.

En Caimanes la fracción fina predomina en el perfil con un promedio de $62,16 \%$, seguida por la fracción intermedia con un valor medio de $21,51 \%$ y esta última no se reporta en la parte inferior. La fracción gruesa no está presente en ambos extremos, pero llega a un promedio de 12,87 $\%$ en los horizontes intermedios.

No se pudo evaluar la distribución granulométrica en la base las cortezas de meteorización ferrosialíticas del sector Farallones y solo se reporta la granulometría de los horizontes superiores. La fracción granulométrica fina predomina 
en estos horizontes y en promedio llega a 90\%, seguido por la fracción intermedia que solo se observa en el horizonte superior con un valor de $13,2 \%$.

En Cayo Guam la fracción fina predomina en todos los horizontes del perfil con un promedio de $52,93 \%$, alcanzando su valor alto $(63,64 \%)$ en el extremo superior. La fracción gruesa tiene un valor promedio de $23,18 \%$ y su valor alto $(45,74 \%)$ se reporta en la parte intermedia del perfil. La fracción intermedia alcanza un valor promedio de $16,02 \%$ y su valor alto $(26,09 \%)$ se reporta hacia la base del perfil.

\section{Caracterización química de los perfiles y cortezas de meteorización ferrosialíticas en Cuba Oriental}

\section{Composición química de las rocas frescas}

Los contenidos de alúmina son relativamente altos en todos los sectores alcanzando concentraciones de hasta $15,73 \%$ en peso. En Cayo Guam la concentración de alúmina es más alto (16,3\% en peso).

Se observan valores altos de la sílice en el rango de rocas básicas a ácidas en toda la región estudiada. En los sectores Baconal y El Culebro estos valores llegan a $68,61 \%$ en peso en las rocas madres. Esto se debe a las alteraciones hidrotermales en estas rocas, evidenciadas por la presencia de vetillas de cuarzo, tanto en las rocas como en las cortezas. En Caimanes y Farallones este componente alcanza valores de hasta $67,54 \%$ en peso.

El contenido de hierro en los sectores estudiados tiene valores entre 1 y $13 \%$ en peso. No se pudo determinar el contenido férrico en las rocas madres del Baconal y El Culebro, pero los contenidos del hierro ferroso llegan a $6,16 \%$ en peso.

Los valores de los álcalis, alcalino-térreos y manganeso no sobrepasan el $10 \%$ en peso. El contenido de magnesio es alto en los gabros de Cayo Guam, llegando a 27,59\% en peso.

Los valores de titanio están entre 0,4 y $0,76 \%$ en peso en toda la región de estudio, siendo más altos en las rocas volcánicas de la Fm. Téneme que en los gabros de afinidad ofiolítica en Cayo Guam.

\section{Valores del pH para los sectores Baconal, EI Culebro, Caimanes y Farallones}

El pH (Cuadro 2) de las cortezas de meteorización aumenta con la profundidad de los perfiles en los sectores Baconal, El Culebro, Caimanes y Farallones, siendo menos ácido en los horizontes inferiores, generalmente. En Baconal, desde la base hasta la superficie (TNL-3 hasta TNL-1) el $\mathrm{pH}$ disminuye en el horizonte intermedio y aumenta de nuevo en la parte superior del perfil, aunque sus valores siempre son menores que 7 .

En El Culebro la parte superior es más ácida que la intermedia y la inferior. Al igual que en el sector anterior sus valores están por debajo del valor de $\mathrm{pH}$ neutro.

En el perfil de Caimanes la acidez aumenta desde la superficie hasta la parte intermedia, de allí tiende a bajar con la profundidad hasta que el $\mathrm{pH}$ alcanza un valor casi neutro en la base.

En Farallones la acidez aumenta con la profundidad y el $\mathrm{pH}$ disminuye en el mismo sentido.

\section{Balance de meteorización a partir de los Índices Químicos de Meteorización}

Los Índices Químicos de Meteorización permitieron la evaluación de la movilidad de los elementos químicos con mayor detalle, la apreciación gráfica de los cambios en la composición química con respecto a la profundidad y la determinación del grado de madurez de las cortezas ferrosialíticas.

Los valores de los índices CIW, CIA y PIA (Cuadro 3) son muy altos en las partes superiores de todos los sectores y muy similares en los sectores Farallones y Cayo Guam, representando una meteorización extrema en todos los sectores, con una tendencia creciente hacia las partes superiores de los perfiles.

El MWPI tiene valores muy pequeños en todos los sectores, evidenciando la pérdida de los álcalis y alcalino-térreos con el avance de la meteorización química. En Caimanes es alto debido los elevados contenidos de los álcalis y alcalino-térreos.

El V tiene valores altos en Farallones y Cayo Guam, mientras que en Caimanes son muy bajos, disminuyendo con el aumento de la profundidad. 
Cuadro 2

Resultados de las mediciones del $\mathrm{pH}$ en las muestras estudiadas

\begin{tabular}{|c|c|c|c|c|c|c|c|}
\hline Sector & Localidad & Muestra & $\mathrm{pH}$ & Sector & Localidad & Muestra & $\mathrm{pH}$ \\
\hline \multirow{7}{*}{ Téneme } & \multirow{3}{*}{ Baconal } & TNL-1 & 5,03 & \multirow{12}{*}{ Farallones } & \multirow{5}{*}{ Caimanes } & TNL-8 & 5,48 \\
\hline & & TNL-2 & 4,56 & & & TNL-9 & 5,41 \\
\hline & & TNL-3 & 6,03 & & & TNL-10 & 4,97 \\
\hline & \multirow{4}{*}{ El Culebro } & TNL-4 & 4,58 & & & TNL-11 & 5,90 \\
\hline & & TNL-5 & 4,85 & & & TNL-12 & 7,07 \\
\hline & & TNL-6 & 6,01 & & \multirow{7}{*}{ Farallones } & \multirow[t]{2}{*}{ TNL-13 } & \multirow[t]{2}{*}{5,21} \\
\hline & & TNL-7 & 5,41 & & & & \\
\hline \multirow{5}{*}{ Cayo Guam } & \multirow{5}{*}{ Cayo Guam } & CG-1 & 4,84 & & & \multirow[t]{2}{*}{ TNL-14 } & \multirow[t]{2}{*}{4,79} \\
\hline & & CG-4 & 4,36 & & & & \\
\hline & & CG-2 & 4,93 & & & \multirow[t]{2}{*}{ TNL-15 } & \multirow[t]{2}{*}{4,5} \\
\hline & & CG-3 & 4,24 & & & & \\
\hline & & CG-5 & 4,28 & & & TNL-16 & 4,54 \\
\hline
\end{tabular}

La R disminuye con la profundidad, sugiriendo una fuerte lixiviación de sílice con respecto al enriquecimiento de aluminio, y esto se refleja en los pequeños valores de este índice en todos los sectores de estudio.

Los Índices de Englund y Jorgensen, M1 y $\mathrm{M} 2$, indican un alto grado de madurez de las cortezas estudiadas.

En Baconal los valores de FL indican que las partes superiores están empobrecidas en potasio y sodio con respecto a la roca madre, pero tienen contenidos elevados de sílice, lo que refleja una lixiviación de los álcalis y alcalino-térreos más intensa que de la sílice. Esta misma situación se evidencia en El Culebro pero los valores indican una lixiviación menos intensa. En ambos sectores los valores del FL tienden a disminuir con el avance de la meteorización.

En Caimanes y Farallones los valores de FL son altos y no mantienen una relación definida con respecto al progreso de la meteorización. Por lo general, este índice tiende a aumentar hacia la roca fresca en Caimanes, pero se observa lo contrario en Farallones. Esto se debe al comportamiento irregular del sodio y potasio en estos sectores y la diferencia en los contenidos de sílice. En Caimanes, los contenidos de potasio, sodio y sílice son mayores que en Farallones, lo que indica que el proceso de lixiviación es más fuerte en Farallones o se trata de una fuente externa de estos elementos en Caimanes. La irregularidad en el comportamiento del FL se puede atribuir también a la toma de irregular de las muestras, efectuado con un desplazamiento tanto lateral como vertical.

En Cayo Guam el FL aumenta a medida que disminuye la profundidad. Esto se debe a una pérdida o lixiviación intensa de la sílice a expensa de los álcalis y alcalino-térreos, cuyos valores se mantienen constantes a lo largo de los perfiles.

El Imob decrece con la profundidad en Baconal. Esto indica que hacia las partes superiores los valores de los elementos móviles se hacen más pequeños, corroborando el FL en este sector. La misma tendencia se observa en El Culebro.

En Caimanes los valores de Imob son alternantemente decrecientes y crecientes hasta llegar a un valor negativo en la parte más inferior del perfil. Este comportamiento variable se debe a las irregularidades en las concentraciones de calcio, sodio y potasio en los diferentes horizontes del perfil. Hay un fuerte enriquecimiento de potasio y un aumento de calcio y sodio en la base del perfil, pero estos elementos se empobrecen bruscamente hacia arriba y su comportamiento resulta 
Cuadro 3

Valores de los Índices de Meteorización Química evaluados en los diferentes sectores de estudio. *MIA derivado del CIA

\begin{tabular}{|c|c|c|c|c|c|c|c|}
\hline Localidad & Muestra & CIW & CIA & *MIA & PIA & WIP & MWPI \\
\hline \multirow[t]{3}{*}{ Baconal } & TNL-1 & 98,77 & 98,41 & 96,82 & 98,77 & 2,10 & 0,83 \\
\hline & TNL-2 & 99,00 & 98,90 & 97,80 & 99,00 & 1,57 & 0,73 \\
\hline & TNL-3 & 66,40 & 64,52 & 29,04 & 65,39 & 37,05 & 9,35 \\
\hline \multirow[t]{4}{*}{ El Culebro } & TNL-4 & 99,46 & 98,20 & 96,40 & 99,45 & 3,72 & 1,16 \\
\hline & TNL-5 & 99,51 & 98,11 & 96,22 & 99,50 & 4,47 & 1,61 \\
\hline & TNL-6 & 99,35 & 94,06 & 88,12 & 99,31 & 12,51 & 3,37 \\
\hline & TNL-7 & 75,51 & 70,71 & 41,42 & 73,72 & 39,07 & 8,07 \\
\hline \multirow[t]{5}{*}{ Caimanes } & TNL-8 & 98,82 & 95,29 & 90,58 & 98,77 & 14,74 & 6,09 \\
\hline & TNL-9 & 99,13 & 95,53 & 91,06 & 99,10 & 16,30 & 7,66 \\
\hline & TNL-10 & 98,78 & 93,64 & 87,28 & 98,71 & 20,18 & 8,31 \\
\hline & TNL-11 & 98,90 & 94,07 & 88,14 & 98,84 & 18,25 & 8,33 \\
\hline & TNL-12 & 86,86 & 67,18 & 34,36 & 81,41 & 66,70 & 12,15 \\
\hline \multirow[t]{4}{*}{ Farallones } & TNL-13 & 99,28 & 99,05 & 98,10 & 99,28 & 2,15 & 1,14 \\
\hline & TNL-14 & 99,51 & 99,40 & 98,80 & 99,51 & 2,10 & 1,19 \\
\hline & TNL-15 & 99,58 & 99,47 & 98,94 & 99,58 & 2,01 & 1,28 \\
\hline & TNL-16 & 99,67 & 99,56 & 99,12 & 99,67 & 2,10 & 1,39 \\
\hline \multirow[t]{5}{*}{ Cayo Guam } & CG-1 & 99,54 & 99,21 & 98,42 & 99,54 & 7,04 & 6,22 \\
\hline & CG-4 & 99,48 & 99,36 & 98,72 & 99,48 & 3,72 & 2,94 \\
\hline & CG-2 & 99,42 & 99,27 & 98,54 & 99,42 & 5,08 & 3,69 \\
\hline & CG-3 & 99,17 & 99,02 & 98,04 & 99,17 & 3,50 & 1,91 \\
\hline & CG-5 & 99,45 & 99,29 & 98,58 & 99,45 & 3,60 & 2,22 \\
\hline \multirow[t]{3}{*}{ Baconal } & TNL-1 & 23,39 & 4,13 & 66,15 & 15,19 & 0,02 & 0,98 \\
\hline & TNL-2 & 22,50 & 4,72 & 93,34 & 24,59 & 0,02 & 0,99 \\
\hline & TNL-3 & 1,39 & 6,27 & 2,27 & 4,01 & 0,61 & 0,47 \\
\hline \multirow[t]{4}{*}{ El Culebro } & TNL-4 & 17,01 & 4,73 & 57,73 & 16,65 & 0,05 & 0,98 \\
\hline & TNL-5 & 12,34 & 4,48 & 56,23 & 12,02 & 0,05 & 0,98 \\
\hline & TNL-6 & 6,76 & 4,68 & 18,29 & 6,42 & 0,18 & 0,93 \\
\hline & TNL-7 & 1,91 & 6,09 & 3,02 & 3,96 & 0,70 & 0,59 \\
\hline \multirow[t]{5}{*}{ Caimanes } & TNL-8 & 4,38 & 2,91 & 24,85 & 4,36 & 0,22 & 0,84 \\
\hline & TNL-9 & 3,12 & 3,16 & 28,40 & 3,05 & 0,20 & 0,86 \\
\hline & TNL-10 & 2,90 & 3,29 & 19,98 & 2,81 & 0,27 & 0,80 \\
\hline & TNL-11 & 2,91 & 3,22 & 21,48 & 2,82 & 0,26 & 0,82 \\
\hline & TNL-12 & 2,24 & 5,38 & 2,97 & 2,22 & 1,40 & $-0,04$ \\
\hline
\end{tabular}


Cuadro 3 (continuación)

Valores de los Índices de Meteorización Química evaluados en los diferentes sectores de estudio. *MIA derivado del CIA

\begin{tabular}{lccccccc}
\hline Localidad & Muestra & CIW & CIA & $*$ MIA & PIA & WIP & MWPI \\
\hline Farallones & TNL-13 & 26,24 & 2,05 & 108,59 & 26,75 & 0,04 & 0,97 \\
& TNL-14 & 24,21 & 2,10 & 173,66 & 23,56 & 0,04 & 0,98 \\
& TNL-15 & 21,81 & 2,16 & 197,58 & 20,75 & 0,02 & 0,98 \\
& TNL-16 & 20,21 & 2,16 & 238,40 & 19,08 & 0,03 & 0,98 \\
Cayo Guam & CG-1 & 8,17 & 0,57 & 143,89 & 7,02 & 1,23 & 0,97 \\
& CG-4 & 20,90 & 0,32 & 162,71 & 21,07 & 1,75 & 0,97 \\
& CG-2 & 10,70 & 1,18 & 149,27 & 10,91 & 0,54 & 0,97 \\
& CG-3 & 19,66 & 1,32 & 105,52 & 21,36 & 0,67 & 0,97 \\
& CG-5 & 14,84 & 1,73 & 148,86 & 15,09 & 0,36 & 0,98 \\
\hline
\end{tabular}

irregular. Puede haber un contacto con una roca o fuente de potasio o pudiera ser un enriquecimiento en minerales potásicos (ortoclasa, microclina, illita). Njila et al. (2010) reportaron la presencia de feldespatos potásicos en estas cortezas. Orozco (2007) reportó la presencia de analcima en las tobas de la zona Caimanes y Farallones.

En Farallones el Imob tiene valores constantes en los horizontes inferiores, lo que indica la poca movilidad de potasio, sodio y calcio entre estos horizontes. En el horizonte superior y los horizontes subyacentes hay una movilidad ligera y un empobrecimiento de estos álcalis y alcalino-térreos hacia arriba a medida que avanza la meteorización.

En Cayo Guam ocurre lo contrario que en Farallones. Los valores son constantes hacia arriba con un enriquecimiento ligero de estos componentes hacia la base del perfil.

\section{Clasificación química de los perfiles de meteorización}

Los materiales en los perfiles de meteorización en los sectores de estudio son ferrosialíticos según sus contenidos de alúmina, hierro y sílice. En algunas muestras tomadas en Farallones y Cayo Guam hay cierta tendencia a la formación de cortezas sialíticas.
Las proporciones de los elementos mayores (Fe: Si: $\mathrm{Al}$ ) en forma de óxidos están comprendidas entre $(1: 5,55: 1,48)$ en Baconal y $(1: 0,40: 2,12)$ en Cayo Guam. Generalmente, los contenidos relativos de alúmina son mayores que $20 \%$ en las partes superiores de los perfiles, disminuyendo a valores muy cercanos a este límite hacia las partes intermedias e inferiores en Baconal y El Culebro; el contenido relativo del hierro (III) en todos los perfiles es menor que 30 $\%$ y la sílice ocupa entre 10 y $70 \%$ de los perfiles en los sectores estudiados.

Estas ferrosialitas se subdividieron en función del Índice de Alitización Potencial (API) y la tendencia hacia la formación de alitas para los sectores de estudio (Fig. 2). Según la escala de Intensidad de Alteración las cortezas en los perfiles de Baconal, El Culebro y Caimanes son ferrosialíticas originadas por procesos de caolinitización; en Farallones y las partes inferiores del perfil de Cayo Guam son materiales ferrosialíticos de alitización ligera. El perfil de meteorización en Cayo Guam se formó a partir de una alitización moderada a fuerte, dando lugar a minerales de aluminio, principalmente la gibbsita. Los minerales arcillosos y de hierro existen de forma subordinada en este perfil. 


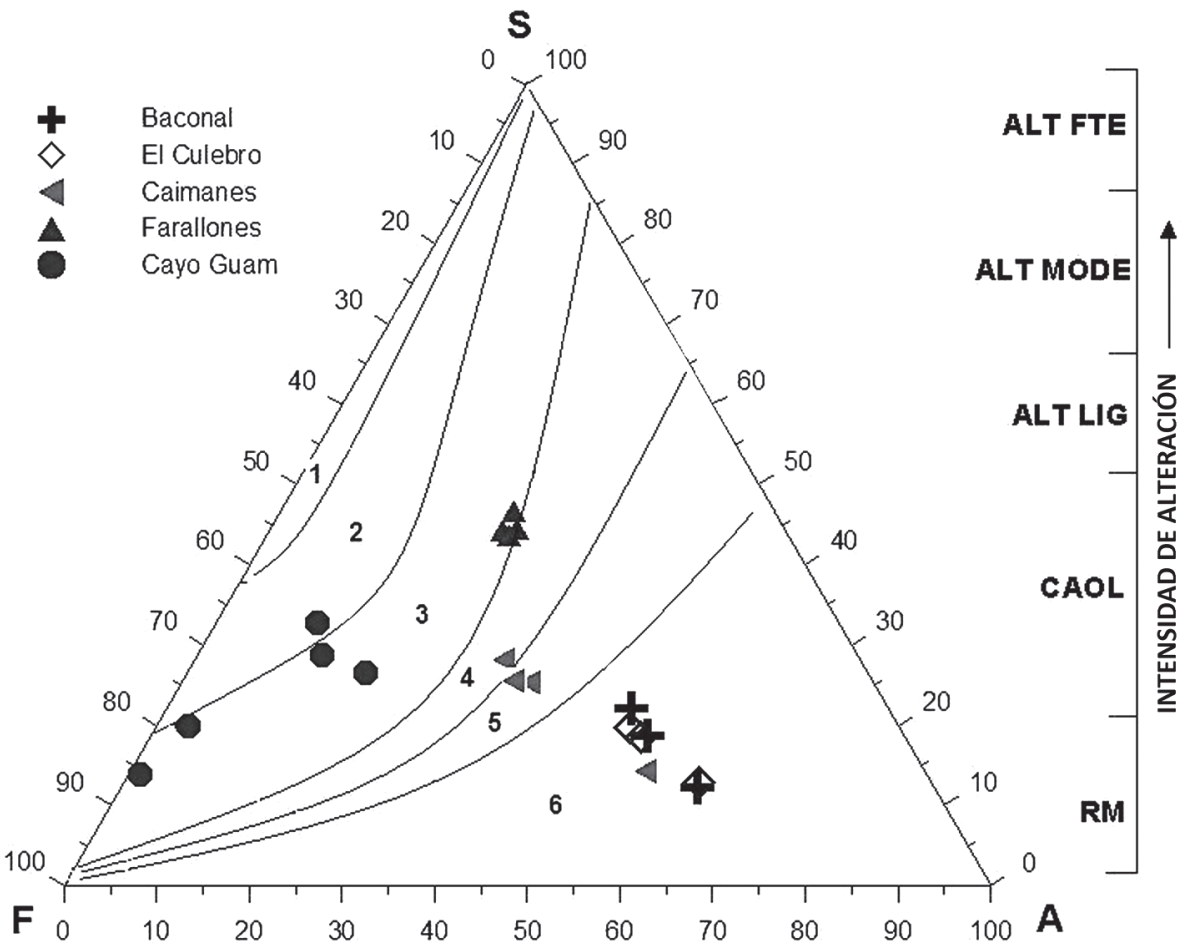

Fig. 2: El Índice de Alitización Potencial en la representación ternaria para la clasificación de productos de meteorización. 1-Bauxitas sensu strictus; 2-Alitas; 3-Arcillas bauxíticas; 4-Arcillas; 5-Materiales arcillosos; 6-Materiales detríticos y no lateríticos.

\section{Caracterización mineralógica de las cortezas y perfiles de cortezas ferrosialíticas en Cuba Oriental}

En Baconal, la caolinita desordenada (Nemecz, 1981) y cuarzo son abundantes en la parte superior del perfil. En la base del perfil aparece la albita.

En El Culebro, la caolinita y el cuarzo predominan en la parte superior del perfil, mientras que la caolinita-montmorillonita aumenta en la parte inferior del perfil. Las vetillas de cuarzo están presentes en las partes inferiores del perfil.

En Caimanes, la hematita predomina en las partes superiores. La halloysita está presente en las partes intermedias y superiores, mientras que la montmorillonita está presente de forma subordinada en la base, donde se concentran el cuarzo y los feldespatos potásicos.
En Farallones las cortezas están compuestas principalmente por caolinita y hematita; la halloysita, montmorillonita y caolinita-montmorillonita aparecen en cantidades pequeñas en las partes intermedias e inferiores.

En la parte superior del perfil de meteorización de Cayo Guam predomina la gibbsita seguida por la halloysita. El análisis mineralógico cuantitativo (Cuadro 4) muestra la abundancia y distribución de las principales fases minerales en cada perfil.

\section{Índices Químico-Mineralógicos}

El diagrama de Abedini et al. (2008) (Fig. 3) representa la clasificación químico-mineralógica de las cortezas de meteorización estudiadas. 
Cuadro 3 (continuación)

Valores de los Índices de Meteorización Química evaluados en los diferentes sectores de estudio. *MIA derivado del CIA

\begin{tabular}{cccccc}
\hline Mineral & Baconal (\%) & El Culebro (\%) & Caimanes (\%) & Farallones (\%) & Cayo Guam (\%) \\
\hline Kln & 46,61 & 35,94 & - & 45,51 & - \\
Gbs & - & - & - & - & 71,63 \\
Gt & - & - & - & - & 2,12 \\
Hem & 2,97 & 0,56 & 52,60 & 30,03 & 1,23 \\
Hyt & - & - & 7,46 & 0,02 & 0,07 \\
Ab & 15,95 & - & - & - & - \\
Q & 34,46 & 49,96 & 19,89 & 0,7 & 0,08 \\
Mnt & - & - & 2,60 & - & - \\
Or & - & - & 17,45 & 24,73 & 19,24 \\
Kln-Mnt & - & 14,04 & - & - & 5,63 \\
Nct & - & - & - & 100,00 & 100,00 \\
\hline TOTAL & 99,99 & 100,00 & 100,00 & & - \\
\hline
\end{tabular}

En Baconal, El Culebro y Farallones los perfiles son de arcillas y están compuestos principalmente por caolinita y montmorillonita en las partes superiores e intermedias pero con la presencia de goethita y hematita. El contenido de los minerales arcillosos aumenta en el orden El Culebro-Baconal-Farallones; en Caimanes son ferritas compuestas principalmente por hematita y goethita con contenidos de minerales de aluminio y titanio muy despreciables y en Cayo Guam son alitas de poco hierro con contenidos altos de minerales de aluminio. Este último perfil está compuesto principalmente por gibbsita con la presencia de caolinita y minerales de hierro en pequeñas cantidades.

\section{Posibles usos industriales con base en la com- posición química}

Se pudo deducir los posibles usos industriales con base en la composición química de los diferentes materiales arcillosos existentes en cada sector estudiado por el diagrama de Avgustinik (1983).

Las cortezas de Baconal y El Culebro son aptas para azulejos de terracota, ladrillos, tubos de calcinación y piezas antiácidas, mientras que las cortezas de Caimanes y Farallones son aptas para ladrillos y pueden ser utilizadas para piezas refractarias. Por el gráfico de Ludwig, se infiere la posible utilidad de las cortezas en Cayo Guam como materia prima para piezas refractarias dada su alta refractariedad.

Además, debido a que estas cortezas están enriquecidas en sílice $(>30 \%)$, todos los materiales detríticos en los sectores referidos pueden tener importancia como materiales aditivos para la producción del cemento Portland y como aditivos a las arcillas refractarias para la pasta de loza empleada en la cerámica.

\section{DISCUSIÓN}

El aluminio, el hierro y la sílice están concentrados en las partes superiores de cada perfil mientras los elementos alcalinos y alcalino-térreos están fuertemente lixiviados. En el Baconal y en El Culebro, el sodio aumenta con la profundidad, probablemente debido a una transformación hidrotermal de la roca madre en un ambiente marino. El titanio muestra valores constantes en todos los perfiles, lo que indica su inmovilidad en estas cortezas.

Las vetillas de cuarzo presentes en los perfiles de Baconal y El Culebro pueden ser relictos de las rocas madres afectadas por extensos procesos hidrotermales, como se plantea en Proenza et al. 


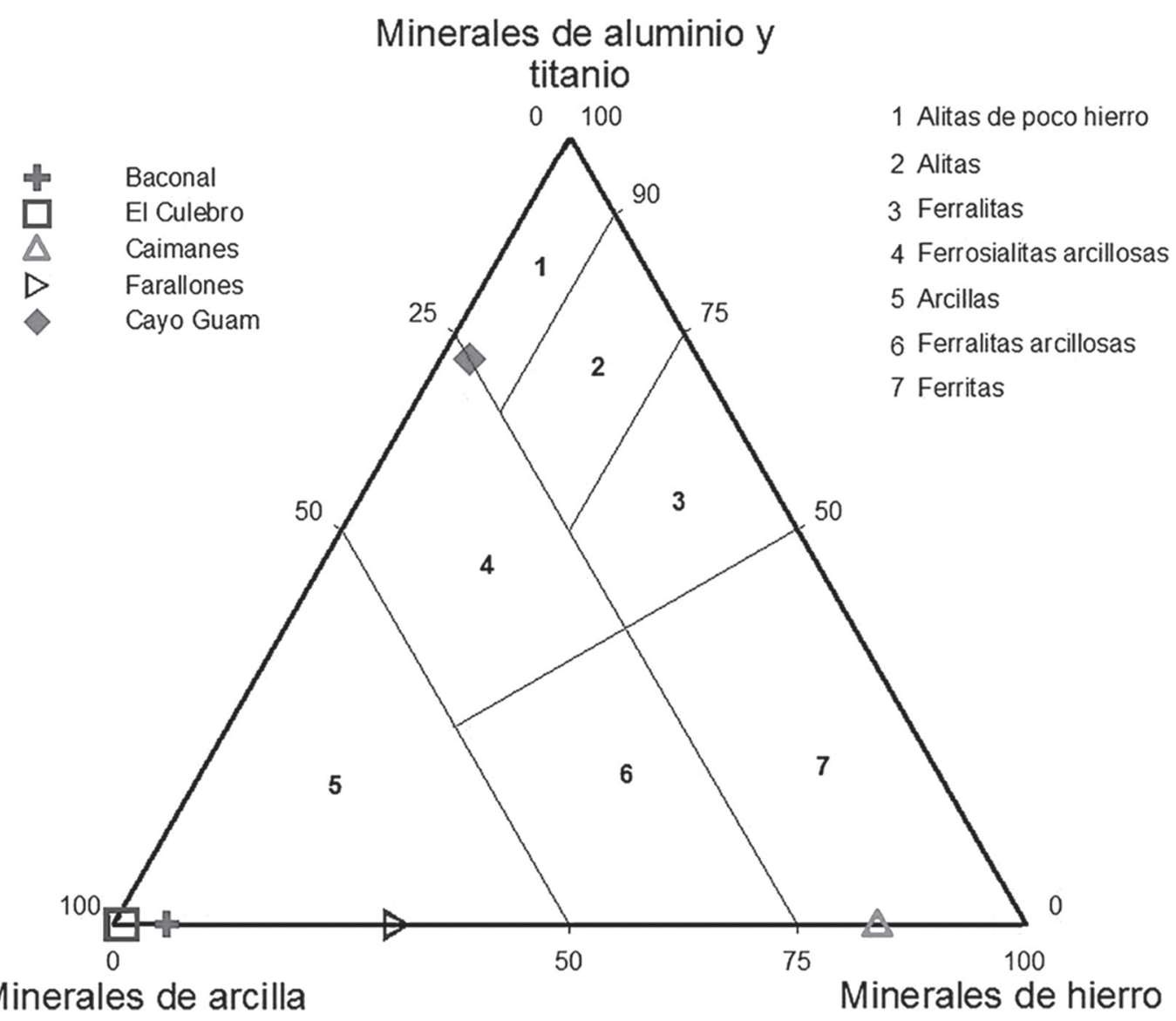

Fig. 3: La clasificación químico-mineralógica con base en el diagrama de Abedini et al. (2008), modificado para este trabajo.

(2006). La cloritización y la albitización pueden haber afectado las partes inferiores de estos perfiles y esto se evidencia por la presencia de esméctitas y plagioclasas (tipo albita).

La presencia de plagioclasas en Baconal sugiere una influencia del intrusivo de Río Grande en las rocas madres de la Fm. Téneme donde se encuentran inclusiones de granodiorita; otra posible explicación de su presencia podría ser la albitización de los volcánicos de esta formación en un ambiente marino.

La halloysita aparece en cantidades variables en Caimanes y Farallones, donde en el primer sector aparece en las partes superiores mientras en el otro aparece en las inferiores.

Los procesos de alteración mineral evidentes en las cortezas estudiadas son la propilitización, albitización, cloritización y la sausuritización de las plagioclasas en los gabros (Gutiérrez, 1982; Torres, 1987).

Generalmente, la composición química y mineralógica, los índices químicos y químico-mineralógicos y los perfiles de Baconal, El Culebro y Caimanes indican que el grado de la transformación de los minerales disminuye hacia la base de los perfiles (ver Cuadro 4, valores de MIA). Estos valores están en el rango de transformación incipiente para las muestras tomadas cerca de la base y intensos a extremos para las muestras de las partes intermedias y superiores. En particular, los valores de los índices químicos y químico-mineralógicos en Farallones son casi constantes en el rango de una transformación mineral extrema.

La investigación desarrollada, hace un importante aporte teórico-práctico a las ciencias geológicas al demostrar por primera vez, la existencia de 
perfiles de meteorización ferrosialíticos desarrollados sobre rocas volcánicas ofiolíticas y vulcanitas de arcos en la región de desarrollo del Arco de Isla Circum-caribeño.

En el orden social, la investigación resuelve de manera rápida y eficiente, uno de los problemas sociales más perentorios que hoy enfrentan los territorios del nordeste de la provincia de Holguín, específicamente Frank País, Sagua de Tánamo y Moa, en relación a la localización y eventual evaluación rápida y efectiva de nuevas fuentes de Rocas y Minerales Industriales como vía alternativa en aras del desarrollo local sostenible.

\section{CONCLUSIONES}

En la práctica, la investigación resuelve de manera satisfactoria los siguientes problemas:

1. Los perfiles lateríticos de Baconal, El Culebro, Caimanes, Farallones y Cayo Guam han sido clasificados en función de su composición química (Fe, Al, Si) como tipo ferrosialítico. En la clasificación por el Índice de Alitización Potencial, en las tres primeras localidades los perfiles están compuestos por materiales arcillosos, detríticos y no lateríticos, con cierta tendencia a la formación de arcillas durante la etapa de caolinitización y el inicio de la alitización en el sector Farallones y en las partes inferiores del perfil de Cayo Guam. En este último algunas partes del perfil llegan a clasificarse como alitas. Por su composición químico-mineralógica los perfiles son arcillosos en los sectores Baconal, El Culebro y Farallones, en Caimanes son ferralíticos y en Cayo Guam son alíticos con poco hierro.

2. Los elementos mayores (Al, Fe, Si) se concentran en las partes superiores de cada perfil. Los álcalis $(\mathrm{Na}$ y $\mathrm{K})$ y alcalino-térreos $(\mathrm{Ca}, \mathrm{Mg})$ son fuertemente lixiviados en las partes intermedias y superiores de todos los perfiles. Sin embargo, el sodio se concentra en Baconal y El Culebro, debido probablemente a los factores hidrológicos y la transformación hidrotermal sufrida por las rocas madres. El titanio muestra un enriquecimiento ligero y su distribución esta en dependencia del grado de madurez de los perfiles.
3. Los perfiles de meteorización en los sectores estudiados están constituidos por gibbsita, caolinita, goethita, hematita, halloysita, montmorillonita, plagioclasas y cuarzo. Por localidad, las asociaciones mineralógicas son: caolinita, hematita, cuarzo y albita (Baconal); caolinita, hematita, cuarzo y montmorillonita (El Culebro); hematita, halloysita, cuarzo, montmorillonita y ortoclasa (Caimanes); caolinita, hematita, halloysita, montmorillonita y caolinita-montmorillonita (Farallones) y gibbsita, goethita, hematita, halloysita, montmorillonita, caolinita-montmorillonita y nacrita (Cayo Guam).

4. El balance de meteorización indica un apreciable grado de madurez de los perfiles de meteorización estudiados. El grado de alitización en los sectores estudiados es muy bajo, excepto en Cayo Guam.

\section{AGRADACIMIENTOS}

Agradecimos al Prof. Dr. Joaquín Proenza Fernández, Universidad de Barcelona, España, por su ayuda en las técnicas analíticas y las sugerencias durante el curso del trabajo, y al Prof. Dr. Hans Gursky, TUC, Alemania, por su ayuda con las técnicas analíticas. Muchas gracias a los colegas Prof. Dr. Antonio Rodríguez Vega, Prof. Dr. Gerardo Orozco Melgar por las revisiones y apoyo.

\section{REFERENCIAS}

AVGUSTINIK, A. I. 1983: Cerámica.- 123 págs. Ed. Reverté S.A., Buenos Aires.

BETANCOURT, Y. 1992: Recopilación científico-técnica y estudio preliminar sobre la cerámica roja en el municipio de Moa y su comparación con el resto de la provincia.- 80 págs. Instituto Superior Minero Metalúrgico de Moa, Cuba [Tesis Lic.].

CÁMARA, M. 2007: Caracterización geoquímica y mineralógica de las cortezas no ferroniquelíferas en Moa, en los ejemplos de 
sectores Centeno y Cayo Guam.- 52 págs. Instituto Superior Minero Metalúrgico de Moa, Cuba [Tesis Lic.].

COBAS, R.M. \& VARGAS, H. 1982: Evaluación de perspectivas bauxiticas en Cuba: la zona de Puriales de Caujerí-Viento Frio, Provincia Guantánamo.- 43 págs. Instituto Superior Minero Metalúrgico de Moa, Cuba [Tesis Lic.].

COBIELLA, J.L. 1978: Estratigrafía y paleogeografía del Paleógeno de Cuba oriental.- 110 págs. Instituto Superior Minero Metalúrgico de Moa, Cuba [Tesis Ph.D].

COUTIN D.P., DUDICH E. \& NAGY, E. 1981: Características de las acumulaciones bauxíticas en la provincia de Guantánamo, Cuba.- 35 págs. Academia de Ciencias de Cuba [Inf. Técnico].

DE DIOS, D. \& CUETO, D. 1982: Evaluación de las perspectivas bauxiticas en Cuba: la corteza de intemperismo en la zona de Quibiján de Baracoa, Provincia Guantánamo.- 56 págs. Instituto Superior Minero Metalúrgico de Moa, Cuba [Tesis Lic.].

ENGLUND, J.O. \& JORGENSEN, P. 1973: A chemical classification system for argillaceous sediments and factors affecting their composition.- Geologiska Foreningens i Stockholm Fohandlingar, 95: 87-97.

FADEL, M. 2005: Evaluación preliminar y caracterización de la manifestación de caolinitas en la zona de Cayo Guam, Moa.- 55 págs. Instituto Superior Minero Metalúrgico de Moa, Cuba [Tesis Lic.].

FEDO, C.M., NESBITT, H.W., YOUNG, G.M. 1995: Unraveling the effects of potassium metasomatism in sedimentary rocks and paleosols, with implications for the paleoweathering conditions and provenance.Geology, 23: 921-924.
FONSECA, D.E., GÓNGORA, E. \& MARTÍNEZ, O. 2004: Evaluación de mezclas de arcillas de la región de Centeno, Moa, Cuba.Ingenierías, 12(22): 45-54.

FONSECA, E.; V. N. ZELEPUGIN y HEREDIA, M. 1985: Structure of the ophiolite association of Cuba.Geotectonic, 19: 321-329.

FORMELL, F. \& BUGUELSKY, Y. 1984: Particularidades de la génesis y evolución del yacimiento Sierra Azul.- Ciencias de la Tierra y del Espacio, 8: 59-69.

FORMELL, F. JAKUS, P. \& GYARMATI, P. 1977: Nuevas áreas perspectivas para la acumulación de bauxitas cársticas en los alrededores de Guantánamo, Cuba. Contribución a la Geología de Cuba Oriental.- 7 págs. Editorial Científico Técnico, La Habana, Cuba.

GARCÍA, M.A. 1985: Materias primas nometálicos para construcción en la provincia Holguín.- 80 págs. Instituto Superior Minero Metalúrgico de Moa, Cuba [Tesis Lic.].

GONZÁLEZ, C.M. 1991: Informe de prospección preliminar y detallada-arcillas para azulejos-Holguín.- 55 págs. Instituto Superior Minero Metalúrgico de Moa, Cuba [Tesis Lic.].

GONZÁLEZ, M. \& BARCAZ, M. 1981: Evaluación de perspectivas bauxiticas en Cuba: Las cortezas de intemperismo en el Quemado del Negro.- 79 págs. Instituto Superior Minero Metalúrgico de Moa, Cuba [Tesis Lic.].

GUERASIMOV, E; GURIEV, N, KOSTAREV, G. \& SAUNDERS, E. 1976: Hallazgo de Bauxitas Estructurales en la Provincia de Oriente.- 76 págs. Editorial Orbe. Moscú. 
GUTIERREZ, M.L. 1982: Estudio geológico y petrográfico de los gabros de Quemado del Negro.- 87 págs. Instituto Superior Minero Metalúrgico de Moa, Cuba [Tesis Lic.].

HARNOIS, L. 1988: The CIW index: a new Chemical Index of Weathering.Sedimentary Geology, 55: 319-322.

HERNÁNDEZ, N. 1989: Procesamiento de la muestra tecnológica $\mathrm{N}^{\mathrm{o}} 12$ del sector Pan de Guajaibón del yacimiento de bauxitas Sierra Azul.- 12 págs. Oficina Nacional de Recursos Minerales, Cuba [Inf. interno].

HERNÁNDEZ, N. \& HERNÁNDEZ, N. 1985: Estudio del tratamiento termoquímico con $\mathrm{NH} 4 \mathrm{Cl}$ de bauxita del Yacimiento Sierra azul.- 22 págs. Oficina Nacional de Recursos Minerales, Cuba [Inf. interno].

IRFAN, T. Y. 1996: Mineralogy, fabric properties and classification of weathered granites in Hong Kong.- Quart. J. Engin. Geology, 29: 5-35.

ITURRALDE-VINENT, M.A. 1998: Sinopsis de la constitución geológica de Cuba.- Acta Geológica Hispana, 33(1-4): 9-56.

ITURRALDE-VINENT, M.A. 1996: Geología de las ofiolitas de Cuba.- En: ITURRALDEVINENT, M.A. (ed.) Ofiolitas y arcos volcánicos de Cuba.- IGCP Project 364 Special Contribution 1: 83-120.

JENNY, H. 1941: Factors of Soil Formation.- 281 págs. McGraw-Hill Press, New York.

LAVANDERO, R.M., BRAVO, F. \& PONCE, N. 2009: Problemática actual de la bauxita y alitas en Cuba.- 8th Congr. Cubano de Geología. La Habana, Cuba: 629-643.

MORENO, B. \& PEREZ, L. 1982: Evaluación de perspectivas bauxiticas en Cuba: Las cortezas de intemperismo en la Gran Tierra.- 52 págs. Instituto Superior Minero Metalúrgico de Moa, Cuba [Tesis Lic.].

NAGY, E., BREZSNYANSZKY, K., BRITO, A., COUTIN, D.P., FORMELL, F., FRANCO, G.L., GYARMATI， P., JAKUS， P. \& RADOCZ, G.Y. 1983: Contribución a la geología de Cuba oriental.- 273 págs. Editorial Científico Técnico, La Habana, Cuba.

NAGY, E., BREZSNYANSZKY, K., BRITO, A., COUTIN, D.P., FORMELL, F., FRANCO, G.L., GYARMATI, P., JAKUS, P. \& RADOCZ, G.Y. 1976: Texto explicativo del mapa geológico de la provincia de Oriente a escala 1:250 000.- 11 págs. Academia de Ciencias de Cuba [Inf. Técnico].

NESBITT, H. W. \& YOUNG G.M. 1982: Early Proterozoic climates and plate motions inferred from major element chemistry of lutites.- Nature 299: 715-717.

NJILA, T., DIAZ-MARTINEZ, R., OROZCO, G. \& ROJAS, A. 2010: An overview of nonnickeliferous weathering crusts in Eastern Cuba.- Minería y Geología, 26 (2): 14-34.

NJILA, T., DIAZ-MARTINEZ, R., PROENZA, J.A. \& OROZCO, G. 2010: Semiquantitative mineralogical evaluation of the non-nickeliferous weathering crusts in Northeast Cuba.- Minería y Geología, 26(4): 1-15.

NJILA， T. \& DIAZ-MARTINEZ， R. 2009: Desarrollo actual de los estudios de diferentes cortezas de meteorización y mineralización asociada a la Faja Ofiolítica MayaríBaracoa.- [resumen] $8^{\text {th }}$ Congr. Cubano de Geología. La Habana, Cuba: 72.

OLIVA, G. 1989: Nuevo Atlas Nacional de Cuba.132 págs. Instituto de Geografía, ACC, La Habana. 
OROZCO, G. 2007: Sobre la presencia de phillipsita y analcima en tobas de la región de Farallones, Moa.- [Resumen] $2^{\text {da }}$ Convención Cubana de Ciencias de la Tierra, La Habana, Cuba, GEO3-P19.

PARKER, A. 1970: An index of weathering for silicate rocks.- Geological Magazine, 107: 501-504.

PROENZA, J.A. \& CARRALERO, N. M. 1994: Un nuevo enfoque sobre la geología de la parte sur de la cuenca de Sagua de Tánamo.- Minería y Geología, 11: 3-10.

REICHE, P. 1943: Graphic representation of chemical weathering.- J. Sedimentary Petrol. 13: 58-68.

REICHE, P. 1950: Survey of weathering processes and products.- 95 págs. Universidad de Nuevo México Publ. Geol. 3, Nuevo México [Inf. interno].

RÍOS, Y.I. \& COBIELLA, J.L. 1984: Estudio preliminar del macizo de gabroides Quesigua de las ofiolitas del este de la provincia de Holguín.- Minería y Geología, 2: 109-132.
ROALDSET, E. 1972: Mineralogy and geochemistry of Quaternary clays in the Numedal Area, southern Norway.- Norsk Geolisk Tidssfrift, 52: 335-369.

RUXTON, B.P. 1968: Measures of the degree of chemical weathering of rocks.- Journal of Geology 76: 518-527.

TORRES, M. 1987: Características de la asociación ofiolítica de la región norte de Moa-Baracoa y su relación con el Arco Volcánico Cretácico.- 113 págs. Instituto Superior Minero Metalúrgico de Moa, Cuba [Tesis Lic.].

VOGEL, D. E. 1975: Precambrian weathering in acid metavolcanic rocks from the Superior Province, Villebon Township, South-central Quebec.- Canadian J. Earth Sci. 12: 2080-2085.

VOGT, T. 1927: Sulitjelmafeltets geologi og petrografi [en noruego, con resumen en ingles]. Norges Geologiske Undersokelse 121: 1-560.

VOICU, G. BARDOUX, M. \& VOICU, D. 1997: Mineralogical norm calculations applied to tropical weathering profiles.Mineralogical Magazine, 61: 185-196. 
